\title{
Le chaos après le déluge : notes sur la crise turque des annèes 70
}

\section{Hamit Bozarslan}

\section{(2) OpenEdition}

1 Journals

Édition électronique

URL : http://journals.openedition.org/conflits/501

DOI : $10.4000 /$ conflits. 501

ISSN : $1777-5345$

Éditeur :

CCLS - Centre d'études sur les conflits lilberté et sécurité, L'Harmattan

Édition imprimée

Date de publication : 15 mai 1997

ISSN : 1157-996X

Référence électronique

Hamit Bozarslan, «Le chaos après le déluge : notes sur la crise turque des annèes 70 », Cultures \& Conflits [En ligne], 24-25 | hiver 1996- printemps 1997, mis en ligne le 27 mars 2007, consulté le 30 mars 2021. URL : http://journals.openedition.org/conflits/501 ; DOI : https://doi.org/10.4000/conflits. 501

Ce document a été généré automatiquement le 30 mars 2021.

Creative Commons License 


\title{
Le chaos après le déluge : notes sur la crise turque des annèes 70
}

\author{
Hamit Bozarslan
}

1 Ingouvernabilité en raison de coalitions parlementaires faibles et souvent contrenature, économie prise dans le cercle infernal inflation-dévaluations-pénuries, brouille avec ses alliés occidentaux à la suite de l'occupation de Chypre (1974) : la Turquie des années soixante-dix additionne les crises. Mais la mémoire collective garde surtout de ces années l'image de la " terreur " et de l'" anarchie ". En effet, entre 1975 et 1980, le pays frôle la guerre civile : les affrontements entre groupes radicaux, de gauche et de droite, font près de 6000 morts. Plus étonnant, la présence étatique disparaît dans de nombreuses localités, y compris dans certains quartiers de grandes villes comme Istanbul. Le système politique est paralysé : l'Assemblée Nationale ne parvient pas, six mois durant, à élire un président de la République, ce qui confirme le vide du pouvoir et aggrave les tensions politiques. En effet, les grandes formations turques, notamment le Parti de la Justice (Adalet Partisi) du conservateur Süleyman Demirel et le Parti Populaire Républicain (Cumuriyet Halk Partisi) du social-démocrate Bülent Ecevit, refusent les concessions qui permettraient de créer des coalitions cohérentes. De plus, en 1980, alors qu'on compte quotidiennement vingt-cinq victimes de la violence politique, la récession économique s'installe et des centaines de milliers d'ouvriers se préparent à lancer une grève générale. Cette période se termine par l'intervention brutale de l'armée, le 12 septembre 1980, et la mise en place d'un nouveau régime politique, qui parvient rapidement à réduire la violence politique au prix d'une répression féroce, notamment contre les militants de gauche ${ }^{1}$.

2 Le paysage politique que nous avons décrit, en le simplifiant considérablement, se rapproche à première vue d'une situation de chaos idéal typique. Pour la population, elle est l'oeuvre $d$ '" ...une force anonyme et incontrôlable qui s'affranchit des déterminations sociales pour être prise en charge en quelque sorte par les entités sociales et les individus les plus divers " 2 . Pour le chercheur, en revanche, la crise turque des années soixante-dix constitue l'occasion de comprendre si, derrière la charge symbolique du terme de chaos, il ne convient pas plutôt de repérer les acteurs 
et les stratégies fluctuantes d'alliances ou d'affrontements. Les processus politiques et sociaux ne sont pas ici l'oeuvre anonyme de structures impersonnelles, qui déboucheraient sur une irrationalité totale. Partant de ce questionnement, on reviendra sur la pertinence d'une " théorie du chaos ", qui fait écho au sentiment de " fin du monde " qui prévalait en Turquie dans les années soixante-dix ${ }^{3}$.

La double fonction de la crise

3 A l'évidence, la Turquie des années soixante-dix passe par une crise multiforme, politique, économique et sociale. Dans un premier temps, on soulignera la fonction heuristique de cette crise pour les sciences sociales. Ce détour méthodologique renvoie l'hypothèse du " chaos " à une phase ultérieure de l'analyse car la crise peut nous permettre de saisir ce qu'il y a derrière la (supposée) perte de sens et le désarroi des acteurs.

4 Pour analyser la crise, partons d'une remarque provocatrice de P. Berger. Ce sociologue explique que : " the fundamental sociological problem is not crime, but law, not divorce but marriage, not racial discrimination but racially defined stratification, not revolution, but government "4. Cette affirmation est largement contestable. Contrairement à Berger, nous tenons que tout acte de " rupture ", ou de " déviance " dans le sens étymologique du terme, constitue un problème fondamental de la recherche sociologique car il accomplit une fonction de révélateur. Il permet notamment de comprendre, a posteriori, quels acteurs et quels mécanismes permettait à l'ordre existant d'être accepté comme légitime, et donc se faire obéir, par un groupe donné. En effet, l'ébranlement de la légitimité et la mise en question de l'obéissance, constitutifs de la crise, ne peuvent être compris que dans leur rapport à la construction de l'ordre social. Autrement dit, les mécanismes liés à la légitimité et à l'obéissance ne deviennent transparents qu'après coup, lorsqu'il y a crise de légitimité et refus d'obéissance. Le fonctionnement social habituel, quand les acteurs agissent dans un champ structuré, est ainsi moins révélateur que les difficultés qu'ils peuvent éprouver dans une période d'incertitude. L'analyse de la crise de légitimité et de la désobéissance peut permettre de saisir, a posteriori, les raisons qui ont permis aux acteurs de s'imposer par le passé comme " légitime " et obtenir l'obéissance et donc, aussi, les raisons pour lesquelles il n'arrivent plus au même résultat. L'" ordre " existant porte en son sein les matrices sociales qui vont ensuite jouer dans la crise et favoriser l'émergence de nouvelles conjonctures.

5 En deuxième lieu, la crise (tout comme la révolution) est un bon exemple de processus dynamique et interactif : elle permet de saisir l'émergence d'acteurs, de passions et de comportements politiques nouveaux. Les répertoires dans lesquels ces derniers s'inscrivent et les jeux qui leur permettent de s'articuler sont inventeurs de nouvelles légitimations, acceptées par tout ou partie de la société. Les crises, et elles seules, donnent à la recherche les situations nécessaires pour comprendre la discontinuité (au sens foucaldien du terme ${ }^{5}$ ), mais aussi la possibilité de penser la continuité de la longue durée. Ainsi, les recherches les plus fructueuses sur le fonctionnement de l'ordre en France (Tocqueville, L'Ancien régime et la Révolution) ou en Russie (M. Malia, Comprendre la Révolution russe) ont été effectuées à partir de l'expérience de la révolution.

La crise comme révélateur de la Turquie des années soixante-dix 
6 Avant d'interpréter les mutations sociales et les ruptures des années soixante-dix, la crise turque nous invite à deux remarques sur le fonctionnement et les mécanismes de légitimation de l'ordre.

7 La première de ces observations concerne la notion d'Etat neo-patrimonial. Issue de la matrice webérienne du patrimonialisme, cette théorie a été appliquée, souvent de façon caricaturale et sans beaucoup d'études empiriques, par de nombreux chercheurs travaillant sur la Turquie (et sur le Moyen-Orient). L'image de l'" Etat-père ", telle qu'elle apparaît dans la culture populaire turque, a été présentée comme un argument central pour démontrer la nature neo-patrimoniale de l'Etat. Or, précisément, la crise des années soixante-dix montre que, si l'idée d'Etat-père impliquait le respect de l'autorité et fournissait une indéniable source de légitimité au pouvoir, elle ne lui assurait pas pour autant une omniprésence ou une force coercitive incontestable. L'Etat fonctionnait, il jouissait d'une légitimité dans le sens où il était souvent obéi hors de la contrainte immédiate. Mais ce succès ne s'expliquait certes pas du fait de sa nature " patrimoniale ". De multiples réseaux informels coexistaient avec l'Etat et échappaient à son contrôle ou, en tout cas, disposaient d'une large autonomie tacitement reconnue. Des associations de divers ordres, rarement politiques dans leur objet ${ }^{6}$, des groupes d'intérêt locaux et des intermédiaires, des notables urbains, des confréries religieuses ou des tribus étaient ainsi intégrés dans le système politique, à une échelle locale, voire nationale. L'Etat reconnaissait tacitement cette autonomie car, leurs intérêts étant essentiellement économiques ou locaux ${ }^{7}$, ces réseaux ne menaçaient pas sa domination de l'espace politique. Par leur rôle quotidien, notamment le règlement et l'arbitrage des conflits ou la redistribution économique, ces acteurs faisaient " aller les choses ", et apportaient donc indirectement une légitimation à l'ensemble du système, en particulier au centre. En deuxième lieu, la présence de ces réseaux déchargeait l'Etat de l'entretien d'un appareil coercitif et administratif coûteux. Les fonctionnaires pouvaient ainsi assurer dans des quartiers " nouvelles villes " (yenisehir), une présence symbolique, et peu coûteuse, de l'Etat ${ }^{8}$. Aucun fonctionnaire, aucun préfet n'était dupe au point de demander l'adhésion profonde aux symboles de légitimation étatique, seule l'absence de contestation était exigée.

8 Il serait cependant naif de donner une image consensuelle de la Turquie de l'époque et d'expliquer l'ordre uniquement par des mécanismes de régulation et d'arbitrage. L'ordre fonctionnait parce qu'il impliquait également un certain nombre de rapports de domination, pour gérer les statuts, arbitrer les conflits sociaux et établir les règles du jeu entre l'Etat et des réseaux largement autonomes. Cela nous amène à une deuxième observation : la régulation du système grâce à un large éventail de moyens -vendetta, promotion des enfants des notables notamment par l'éducation ou encore attribution du statut de most favored lords à quelques propriétaires fonciers- s'avérait possible tant que les ressources économiques et politiques restaient limitées et stables. L'allocation des ressources devenait périlleuse dans une situation de transformation rapide marquée par l'intégration dans le système économique international, l'exode rural, l'immigration vers l'Europe, et l'explosion des moyens de communication. Dans le cadre un peu illusoire d'un paysage politique bipolaire', ces mutations ont produit inévitablement de nouveaux acteurs socio-économiques, qui établissaient leur propres clientèles et remettaient en cause la redistribution existante des richesses. Dès les années soixante, ces acteurs, dans la plupart des cas provinciaux ou d'origine provinciale, ont démontré leur capacité de mobilisation en entrant dans la compétition 
politique, parfois en optant pour des partis marginaux. Dès lors, l'action de ces acteurs, pourtant vitale pour la survie du système, ne manque pas de fragiliser le " centre " et de renforcer la place du " local " au détriment du " national ".

9 Ainsi, la symbolique valorisant l'obéissance et le " respect de l'Etat " (mais impliquant aussi la distance à son égard) coexistait avec la priorité accordée aux enjeux locaux. Les enquêtes des années soixante et soixante-dix montrent clairement que la méfiance envers l'Etat était à son paroxysme, alors que le contournement de l'Etat par jeu de réseaux, voire l'acceptation de l'illégalité, s'exprimait au grand jour ${ }^{10}$. Cette attitude n'implique pas une révolte ouverte, on est bien loin d'une situation prérévolutionnaire, ni même une contestation coordonnée du pouvoir. Mais cette méfiance généralisée se fait sentir même au sein de la population turco-sunnite qui, traditionnellement, assure une base sociale à l'Etat. De façon significative, l'Etat était respecté, mais ne jouissait pas pour autant d'une véritable légitimité en tant qu'institution représentant l'ensemble de la société. Il était obéi car il s'imposait comme un arbitre habile, capable de négocier la promotion et la coercition et, surtout, de composer avec la complexité et la multiplicité des provinces anatoliennes. Le bouleversement de ces équilibres anciens, qui s'effritaient dès les années soixante comme le montrent certains signes avant-coureurs, s'est traduit par l'émergence d'acteurs nouveaux, la radicalisation des anciens et la militarisation des dynamiques centrifuges, c'est à dire par une grave crise sociale et politique.

La crise comme processus dynamique et interactif

10 C'est là, dans cet ordre d'" avant " que se trouvent les germes de la crise des années soixante-dix. En effet, à partir des années soixante, cette gestion de la complexité devient difficile en raison des mutations sociales. Les rapports de domination, entre groupes sociaux et par rapport à l'Etat, ne sont plus admis comme allant de soi et cette remise en cause concerne tous les échelons du pouvoir et de la société. Du côté des institutions, l'armée garde son rôle d'arbitre de la vie politique, mais n'en perd pas moins une partie importante des prérogatives qu'elle possédait auparavant. De même, les deux pôles traditionnels du système politique, conservateur et social-démocrate, éclatent, en partie sous le coup des différenciations socio-économiques en cours et de la naissance de nouveaux groupes d'intérêt. Les deux grandes formations, le Parti de la Justice (Adalet Partisi et le Parti Populaire Républicain (Cumuriyet Halk Partisi), connaissent d'importantes scissions qui les fragilisent et déclenchent un processus de fragmentation du système politique. En dehors du jeu institutionnel établi, des formations politiques émergent, peu durables mais néanmoins représentatives, comme le YTP (Parti de la Turquie nouvelle), le TBP (Partie d'Union de la Turquie) ou le TIP (Parti Ouvrier de la Turquie), qui obtiennent des scores conséquents dans certaines provinces anatoliennes. Leur succès tient à l'émancipation d'acteurs qui jusque là se contentaient d'un champ de manoeuvre octroyé par le pouvoir et demandent désormais plus de liberté d'action. Cette demande d'autonomie est une politisation, dans le sens où le partage des biens politiques devient désormais la condition sine qua non du renouvellement du contrat tacite entre de nombreux groupes sociaux et le centre. Mais les difficultés que posent ces demandes ne sont pas uniquement liées aux rapports de force avec le centre. L'ordre hiérarchique interne de nombreuses provinces et des grandes métropoles, dont l'espace urbain et la démographie connaissent des mutations vertigineuses, est également en crise. L'accès au politique, et donc à la citoyenneté, se fait, comme l'avait remarqué Jean Leca dans un autre contexte, au prix 
de la " civilité "11 car les acteurs veulent monopoliser la représentation et les ressources politiques, au prix de la marginalisation des autres groupes.

11 C'est dire que, au seuil des années soixante-dix, les acteurs provinciaux n'acceptent plus leur rôle traditionnel, alors que se constituent à la marge de nouveaux acteurs : classe ouvrière, population des bidonvilles, jeunesse estudiantine, dont l'intégration dans le système politique constitue la revendication principale. La structure politique bipolaire, issue des années cinquante et arbitrée (au moins à l'origine) par l'armée, est mal préparée à cette évolution. Malgré les coups d'Etat de 1960 et 1971, le système avait pu accepter l'opposition libérale/conservatrice et le glissement du parti de Mustafa Kemal vers la social-démocratie ${ }^{12}$, mais il se montre désormais incapable d'intégrer de nouvelles forces politiques. Par ailleurs, si les nouveaux acteurs ne sont pas forcément dépourvus d'expérience politique, ils n'ont pas pour autant les moyens nécessaires pour réaliser leur intégration " pacifique ", c'est à dire contourner les partis en place et instaurer de nouveaux rapports de domination. Cette faiblesse explique largement que certains d'entre eux aient tenté de monopoliser l'espace politique pour s'imposer comme " acteur unique ", voire à le faire complètement éclater afin d'ériger du neuf sur ses ruines.

Quelles sont ces forces nouvelles qui tentent de s'intégrer dans le jeu politique ? Partant des informations disponibles, nombreuses quoique éparpillées, nous pourrions certes essayer de les nommer, ou du moins de les distinguer à partir de leur appartenance doctrinale ${ }^{13}$. Une telle tentative de classification a d'ailleurs son intérêt pour l'histoire politique de la Turquie. Elle serait cependant peu utile pour notre propos car elle risquerait de faire perdre de vue la fragilité des frontières " idéologiques " qui séparent ces acteurs, ainsi que les nombreuses interactions qui les lient. Plutôt que de les énumérer, nous essayerons donc de saisir les conditions de leur émergence et leur logiques d'action.

13 Ces forces sont, en premier lieu, nées de la politisation des provinces qui jusque là étaient restées silencieuses ou avaient cautionné l'une des deux formations majeures du pays, le Parti Populaire Républicain et le Parti de Justice. Dans les années soixante, les provinces ${ }^{14}$ avaient connu une première expérience politique, mais la mobilisation ne dépassait guère les campagnes électorales ou, parfois, les manifestations de rue. Les mutations socio-économiques, notamment l'industrialisation et la généralisation des moyens de communication (radios et télévision) entraînent ensuite une politisation accélérée qui se traduit par une mobilisation quasiment ininterrompue. En particulier, toutes les villes de province connaissent un exode rural important qui bouleverse les rapports de domination entre le monde urbain et rural. Les notables urbains, jusque là seuls habilités à représenter politiquement la localité et à gérer les rapports intercommunautaires, voient leur pouvoir contesté. De plus, l'exode rural accroît considérablement l'importance du chef lieu du département qui devient, plus que par le passé, le centre névralgique de la vie provinciale, marginalisant de fait les groupes qui en sont éloignés. De nouveaux acteurs, qui mobilisent notamment sur une base segmentaire, investissent le milieu urbain et cherchent à en monopoliser les ressources économiques, entreprises publiques et fonctionnariat, et politiques, notamment le contrôle de la municipalité et la représentation à l'Assemblée nationale. Cette concurrence porte à son paroxysme les tensions entre les communautés kurdes et turques, alévies et sunnites. Les modes de règlement traditionnels comme la vendetta s'avèrent désormais incapables de rétablir les équilibres passés. 

une rupture de continuité "17, rendue possible par l'explosion des médias et par la toute récente socialisation urbaine de la deuxième génération immigrée. Dans la plupart des cas, les générations nées dans les années cinquante arrivent à l'âge adulte dans des centres urbains et les jeunes étudiants (voire lycéens et collégiens ensuite) manifestent leur volonté de s'imposer comme acteurs politiques. Dans les grandes métropoles, mais aussi en province, les jeunes s'imposent comme des acteurs dynamiques de l'espace politique. Pour ce faire ils disposent de deux atouts : les " générations aînées " ou " assagies ", notamment celle de leurs parents, leur accordent une sorte de " moratoire psychosocial "18 avant d'être complètement dépassées par leur activisme. En second lieu, des groupes politiques, se réclamant de doctrines radicales de droite ou de gauche mais rarement islamiste, instrumentalisent leur action, mais leur permettent néanmoins d'accéder au statut d'acteur politique.

17 La crise politique turque des années soixante-dix se produit donc à la jonction de plusieurs dynamiques engendrées par ces mutations socio-économiques et ne peut se réduire à une seule dimension ou à une causalité unique, et certainement pas à un phénomène $d$ " anomie ". De façon indéniable, un décalage demeure entre la réalité de ces dynamiques, déjà décelables bien avant la crise ouverte, et la perception de la population qui vit cette situation comme chaotique, voire apocalyptique. Cependant, on est bien loin du chaos, même si les acteurs sont incapables de maîtriser complètement le jeu. Le processus de crise permet, au contraire, la mise en oeuvre de diverses stratégies collectives et la formulation de nombreuses revendications, d'autant plus que de nouveaux acteurs émergent dans la crise ${ }^{19}$. Ces groupes oeuvrent simultanément et en interaction, leurs stratégies peuvent donc être observées et comprises par leurs 
effets sur les autres groupes. Le passage de l'un des acteurs à l'action politique ou, comme nous le verrons, militaire influe directement sur la stratégie de l'ensemble des protagonistes. La contestation violente, par un seul, suffit à remettre en cause la légitimité de l'ordre ou, du moins, à banaliser la perspective de monopoliser le champ politique par la violence. Les acteurs restés jusque-là passifs, indécis ou cantonnés à l'action politique traditionnelle ne peuvent pas rester hors de ces confrontations. Contraints par le jeu d'interactions, les acteurs " respectables ", notamment les deux grandes formations, se prêtent localement, voire dans certains cas à l'échelle nationale, à des alliances avec certains groupes armés ou radicaux.

En raison de cette pluralité des mobilisations, la dichotomie " droite-gauche " dans laquelle les acteurs s'inscrivent, est largement inopérante, en tout cas insuffisante pour expliquer les dynamiques de la crise. En deuxième lieu, les réponses des acteurs à la crise ne peuvent pas non plus être comprises à partir d'un schéma unique. En effet, derrière la violence au quotidien se dessinent différentes trajectoires individuelles ${ }^{20} \mathrm{et}$, principalement, collectives. Ainsi, en profitant de l'ingouvernabilité du pays, un parti islamiste, le MSP (Milli Selamet Partisi) ${ }^{21}$, parvient à s'intégrer pacifiquement dans le système politique pour en devenir une composante durable. Comptant sur l'appui d'une partie de la bourgeoisie et des notables des provinces sunnites, où il trouve son origine, ce parti a tout intérêt à prouver qu'il a les moyens économiques et financiers de réussir son intégration. Pour les autres acteurs, la réponse à la crise passe par la violence qui prend l'Etat pour cible ou vise à le contrôler au détriment des acteurs concurrents. Finalement, la crise donne naissance à cinq stratégies de passage à la violence.

19 Les groupes révolutionnaires de gauche, déchirés entre eux, et ceux de la droite radicale, comme le MHP (Parti d'Action Nationaliste), parviennent à créer des structures centralisées. Leur stratégie de conquête du pouvoir privilégie l'action dans la rue $^{22}$ et la violence massive exercée sur les " adversaires " par des militants entraînés dans des organisations paramilitaires. Cette stratégie passe par différentes modalités d'action : conflit frontal avec l'Etat ou recours à la violence au nom de la survie de l'Etat et de la nation. Cependant, quelle que soit la logique et les motifs invoqués pour recourir à la violence, ces acteurs oeuvrent pour leur propre compte, en dehors de l'Etat. Sans en être la cause ultime, les " doctrines " radicales, qu'elles soient de gauche ou de droite, alimentent la violence et constituent la grille de lecture qui fournit les " justifications normatives " nécessaires aux acteurs ${ }^{23}$.

20 Les provinces " mixtes " (alévies-sunnites) et kurdes, ainsi que certains bidonvilles (gecekondu), sont travaillés par des tensions inter-communautaires et connaissent une politisation accélérée qui favorise le passage à une violence de type milicien. Les groupes politico-militaires issus de différents segments investissent les centres urbains, notamment par le contrôle des municipalités et par une présence militaire dans la ville. La création de " tours de contrôle " (lieux " stratégiques " contrôlant l'accès de rues, voire de quartiers) et la coercition externe (contre la population " adverse ") ou interne (dans son propre groupe) permettent l'instauration d'un contre-pouvoir qui cherche le contrôle des biens culturels, de la taxation économique, du recrutement par le biais de la protection, et de l'administration de la justice. La municipalité, en tant que centre de décision et de légitimation, devient le pivot de ce système milicien qui paralyse, voire chasse, l'Etat et marginalise le segment adverse ${ }^{24}$.

21 La jeunesse, notamment estudiantine, est divisée entre deux groupes radicaux, les " loups gris " ou " idéalistes " (de droite) et les " révolutionnaires " (de gauche). Au-delà 
de cette polarisation, on peut cependant interpréter l'engagement de la jeunesse comme le produit d'un conflit de générations dû à son émergence comme acteur social et politique qui affirme ses propres solidarités. La mobilisation de la jeunesse s'explique essentiellement par la volonté de gagner une légitimité et une reconnaissance ${ }^{25}$, bien qu'en pratique l'engagement conduise à la violence dans les groupes miliciens ou dans les structures politiques centralisées, comme le MHP ou les groupes révolutionnaires. Par ailleurs, le mouvement ouvrier connaît une remarquable mobilisation dans cette période et produit également des formes d'action violente qui dépassent le cadre syndical classique. Le mouvement ouvrier prend aussi en compte les questions sociales comme le problème des gecekondu et les revendications collectives pour la défense des lots de terrain construits, contre la cherté de la vie etc...Ces mobilisations, qui n'ont pas été étudiées comme elles le mériteraient, fournissent un terrain d'action aux groupes de gauche. La paysannerie, dans une moindre mesure, utilise également des formes d'action violentes dont enjeu est la réforme agraire ou l'occupation des terres.

Enfin, sous le coup de ces diverses contestations, les contradictions internes de l'Etat, qui est loin de constituer un bloc monolithique, se sont considérablement intensifiées, aboutissant à deux effets contradictoires. D'une part les groupes politiques sont parvenus à s'intégrer dans certaines instances de décision du pouvoir, notamment la droite radicale pour la police. D'autre part, l'Etat, réduit à sa composante coercitive (police et armée), s'est trouvé de plus en plus impliqué dans l'engrenage amenant le coup d'Etat de septembre 1980.

Ces divers types d'actions violentes ont pour dénominateur commun l'incapacité à recomposer pacifiquement l'espace politique et, plus généralement, l'espace urbain. Ainsi s'explique la tendance à monopoliser ces espaces, caractéristique de la plupart des acteurs. Néanmoins, les actions violentes continuent de s'inscrire dans des répertoires distincts : les nouveaux groupes politiques, pour peu qu'ils soient dotés de structures centralisées, ont une stratégie de conquête du pouvoir à l'échelle de la Turquie. L'éclatement interne de la " gauche " l'empêche de se transformer en acteur unifié, mais le MHP (le Parti d'Action Nationaliste), parfois allié à certaines autorités comme la police ou l'armée, s'est doté des moyens de ses ambitions. La jeunesse estudiantine, nous l'avons souligné, se reconnaît dans ces stratégies, même si son action est instrumentalisée par les partis. De la même façon, la classe ouvrière, éclatée entre plusieurs confédérations, ne parvient pas à développer une action autonome et s'inscrit, généralement à son corps défendant, dans cette logique d'affrontement. Pour sa part, l'action milicienne ne vise pas à conquérir le pouvoir national, mais à monopoliser l'espace politique local, en imposant ce fait accompli au centre. En ce sens, la conquête d'une municipalité par la voie électorale, est porteuse d'une légitimité populaire qui renforce l'autonomie locale acquise par la violence. Mais la coexistence de ces différents enjeux et les stratégies de la violence fragilisent le centre et font peser une menace de guerre civile.

Le chaos ou l'autonomisation de la violence

25 Après cette analyse rapide de la crise des années soixante-dix, nous pouvons à présent revenir à l'hypothèse de la perte de sens et de repères, le " chaos ", qui veut que les processus déclenchés par les acteurs s'autonomisent et se perpétuent de façon autonome, sans intervention des acteurs initiaux, ou même en dépit d'eux. Les acteurs deviendraient ainsi les agents exécutant d'un dessein conçu hors de leur volonté. A la 
limite, le paradigme du chaos s'apparente plus au millénarisme qu'aux problématiques des sciences sociales.

Certes, à observer la Turquie des années soixante-dix, force est de reconnaitre que les processus déclenchés, notamment la violence dans toute sa diversité, gagnent une autonomie par rapport aux acteurs, mais cela ne signifie pas pour autant l'indifférenciation des acteurs dans un désordre généralisé. Dans les faits, la situation est plus simple : à un moment donné de la crise, les acteurs ne parviennent plus à maitriser l'interaction de leurs stratégies. Des éléments multiples font qu'il devient difficile pour eux d'en finir avec la violence, en raison notamment de l'autonomie des acteurs locaux, qui ne répondent pas de leurs actions devant une autorité centrale, et des surenchères, le mythe des martyrs faisant de toute concession une trahison.

Peut-on pour autant parler de chaos ? L'analyse de la crise des années soixante-dix pousse à répondre par la négative. Tout d'abord, l'impossibilité de maîtriser la situation à l'échelle nationale ne signifie pas que les acteurs ne gardent pas localement la situation en main. La situation des provinces qui constituaient le théâtre de la violence milicienne permet, au contraire, de voir que les acteurs locaux sont restés maitres de leur terrain jusqu'à la nuit du coup d'Etat. Pour la population de ces provinces, la situation était devenue effectivement insupportable dès les élections législatives de $1977^{26}$, car la violence des groupes armés, notamment de droite, prenait de plus en plus pour cibles les non-combattants, dépourvus de toute influence sur le jeu politique. Pour les groupes organisés, cependant, l'action continuait d'avoir un sens. L'autonomie d'une province ou du quartier d'une grande ville était gagnée grâce à la violence contre l'Etat ou un autre groupe social ou politique. Il était donc naturel qu'elle se perpétue par la mise en place de mécanismes de plus en plus coercitifs. La terreur contre les civils, loin d'être aveugle, était une manière efficace d'homogénéiser la population, de faire accepter la domination du groupe et d'en imposer les pratiques (justice, recrutement, taxation, contrôle des biens culturels). C'était, en tout cas, une stratégie tout à fait assumée ${ }^{27}$. Par ailleurs, rien n'indique que les acteurs aient tous perdu la maitrise de la situation nationale. La droite radicale, par exemple, a opté délibérément pour une stratégie violente, la seule qui, à son sens, lui donnait les gains refusés par les urnes. Il en allait de même pour certains groupes de gauche comme le PKK (Parti Ouvrier du Kurdistan) dont le chef, Abdullah Öcalan, considérait cette période comme le prélude à un combat de plus grande envergure. Le fait que la nuit du 11 au 12 septembre 1980 ces stratégies de violences, toutes catégories confondues - tout comme les actions pacifiques d'ailleurs ${ }^{28}$ - se soient soldées par un échec, ne peut guère être compris comme le symptôme d'absence de sens.

De plus, les actions des acteurs de cette époque ont produit des effets que sans doute ils ne visaient pas consciemment mais qui, dans la durée, sont à l'origine des matrices politiques actuelles, et donc de sens. Le paysage politique et social de la Turquie actuelle s'est en partie constitué à ce moment. Il est vrai que le mouvement ouvrier a disparu avec la crise, en tout cas sous cette forme, mais la plupart des organisations politiques qui ont vu le jour ou se sont imposées dans les années soixante-dix sont aujourd'hui opérationnelles. Le PKK et le MHP ont survécu au coup d'Etat, mais aussi des groupes de gauche, en particulier ceux qui recrutent dans la communauté alévie. Ces derniers sont actifs actuellement dans les bidonvilles alévis d'Istanbul, comme l'ont montré les scènes d'émeutes de mars 1995. De même, les dynamiques segmentaires, à l'origine du phénomène milicien, ont donné naissance à des acteurs locaux qui 
aujourd'hui se trouvent aux commandes de nombreuses villes de l'Anatolie. Ces nouveaux venus ont réalisé au moins l'un de leurs objectifs : faire accepter leur autonomie par le centre, autrement dit s'intégrer au système aux conditions qu'ils avaient fixées. Ils ont également pu asseoir les mécanismes de clientélisme qu'ils avaient installés. En ce sens on peut difficilement parler d'une déstructuration chaotique de l'" ordre " mais, au contraire, d'une construction qui s'inscrit dans la durée. De même, les nouveaux découpages territoriaux, dessinés en Anatolie sous l'action des groupes miliciens, ont perduré. Là encore, il n'est pas aberrant de parler de construction de nouveaux rapports de domination.

Toutes ces raisons invitent à relativiser la victoire des militaires le 12 septembre 1980 : l'armée a certes montré qu'à ce stade du conflit, les populations civiles, y compris celles qui apportaient un soutien aux groupes armés, pouvaient accepter la coercition venue d'une instance perçue comme impartiale. Mais cette intervention montrait aussi que l'Etat ne pouvait avoir de légitimité que réduite, sans prétention au monopole de la scène politique. Sa domination, rétablie au prix d'une coercition massive entre 1980 et 1983, ne signifie pas que les constructions des années soixante-dix pouvaient être facilement démantelées. Aujourd'hui, si l'Etat se maintient dans de nombreuses localités qui ont été le théâtre de violences dans les années soixante-dix, la raison est qu'il a de facto accepté leur autonomie, quitte à en devenir le garant par exemple en acceptant une militarisation de certains segments sunnites de la population. l'Etat est devenu l'acteur majeur de la scène politique, mais il n'est qu'un acteur. L'image de " l'Etat-père " a cédé la place à celle de l'" Etat qui peut nous trahir "29.

Trois remarques en guise de conclusion

30 La logique de notre analyse amène trois remarques conclusives. En premier lieu, qu'il soit permis de dire la gêne que suscite, à deux titres, l'hypothèse du chaos. Celle-ci s'apparente volens nolens à la théorie de l'irrationalité, qui puise dans les théories leboniennes du tournant $d u$ siècle ${ }^{30}$ et finit inévitablement par prendre l'anomie comme grille d'interprétation. Elle souffre aussi d'un deuxième handicap : les perceptions développées dans des situations de violence ne sont pas reconnue comme une catégorie symbolique, comme un objet d'étude méritant effectivement une analyse approfondie, mais comme un paradigme sociologique. Ainsi le chercheur, fusionnant avec son objet, s'interdit à jamais son analyse.

31 La deuxième conclusion est que la crise turque ne se réduit pas à un désordre, encore moins à l'anarchie comme l'entendait le sens commun turc. La crise n'éclate pas, comme on l'a parfois affirmé, brusquement, et absurdement, avec l'émergence d'une gauche ou d'une droite radicales, en réalité présentes depuis soixante-dix ans. Elle ne se termine pas, non plus, avec l'occupation de l'espace politique par les militaires, qui contraint les autres acteurs au retrait. Le processus de crise devient intelligible comme résultante des stratégies fluctuantes, parce qu'adaptatrices, des différents acteurs en interaction. L'imprévisibilité des actions sociales tient à ce que l'interaction entre ces acteurs ne peut être lue dans un code génétique, 1975 n'augure pas fatalement 1980 ! Ici comme ailleurs, les choses auraient pu se dérouler différemment. L'imprévisibilité des événements ne s'explique pas par l'inconsistance ou la panique des acteurs : à défaut d'une articulation concertée et consentie entre plusieurs stratégies, c'est l'interaction non maîtrisée entre elles qui transforme le paysage politique et social. C'est d'ailleurs ce point qu'il importe d'analyser : sous quelles formes, selon quelles modalités et à quel moment, ces interactions ont-elles eu lieu ? Quelle est la capacité des acteurs à 
s'adapter à ces nouveaux paysages, à réajuster leurs stratégies, à peser sur les processus qu'ils déclenchent? La réponse à ces questions ne peut être trouvée qu'en admettant l'historicité de chaque crise. La recherche, pour être fructueuse, doit convenir, une fois pour toutes, que toute généalogie de l'action sociale est nécessairement complexe et s'inscrit dans un processus dynamique. Il faut refuser l'idée d'une causalité et d'une trajectoire uniques qui détermineraient totalement un processus social ${ }^{31}$, avec des situations chaotiques comme seule alternative.

Enfin, il nous semble important de nous intéresser à ce qui se dessine derrière la crise et, en nous plaçant dans la longue durée, de saisir les " unitended conséquences " de l'oeuvre des acteurs comme nous l'invite à le faire P. Berger ${ }^{32}$. Or, vu sous cet angle, l'éclatement engendré par la crise turque entraîne une réinvention des espaces sociaux. Il n'y a pas eu de fragmentation à l'infini du social et de l'espace politique, au contraire, quelles que soient leurs intentions, les acteurs ont construit des espaces homogènes, susceptibles de leur garantir un champ de manoeuvre suffisamment large. Les espaces locaux n'ont pas proliféré, mais se sont souvent articulés à l'espace national, sous des formes nouvelles et durables. La crise des années soixante-dix n'a donc guère été l'oeuvre de démolition tous azimuts d'acteurs inconscients. Parallèlement à la déconstruction violente du social à laquelle ils ont procédé, ces acteurs, nouveaux pour la plupart, ont reconstruit le jeu social. Sans nier l'importance de leurs récit comme source historique ou de leurs déclarations d'intentions, et sans nullement sous-estimer l'horreur qu'inspire l'expérience de ces années noires, ce sont, à notre sens, ces reconstructions qui devraient principalement mériter notre attention.

\section{NOTES}

1.Cf. notamment, Cevdet Arsan, " Terörün Psikolojik Nedenleri ve Çözüm Yollari ", in Gazeteciler Cemiyeti, Türkiye'de Terör. Abdi Ipekçi Semineri, Istanbul, Gazeteciler Cemiyeti, 1979, p. 182 ; Dogu Ergil, Türkiye'de Terör ve Siddet, Ankara, Turhan Kitabevi, 1980, S. Vaner, " Violence politique et terrorisme en Turquie ", Esprit, oct-nov. 1984, pp. 79-104 ; I. Cem, articles réunis dans son Siyaset Yazilari, 1975-1980 Türkiyesi, Istanbul, Cem Yayinlari, 1980 ; S. Mardin, " Türkiye'de Gençlik ve Siddet ", in S. Mardin, Türk Modernlesmesi, Makaleler 4, Istanbul, Iletisim yayinlari, 1991, pp. 251-290 ; Ömer Laçiner, notamment, " Türkiye'de... Maras'tan Sonra ", Birikim, nº6-47, 1978-1979 et Jean-François Bayart, " La question Alevî dans la Turquie moderne ", in O. Carré (dir.), L'Islam et l'Etat dans le monde d'aujourd'hui, Paris, PUF, 1982, p. 109-120. Un seul ouvrage, celui de Tanil Bora et Kemal Can revient sur cette période pour analyser la trajectoire de l'extrême droite, Devlet, Ocak, Dergâh. 12 Eylül'den 1990'lara Ülkücü Hareket, Istanbul, Iletisim, 1991.

2.D. Pecault, " Violence et politique : quatre éléments de réflexion à propos de la Colombie ", Cultures \& Conflits, n¹3-14, 1994, p. 157.

3.Au-delà, c'est tout le contexte intellectuel de l'élaboration de cette problématique qui semble être en cause : la crise turque datait bel et bien de l'époque de la guerre froide, mais elle lui a survécu parce qu'elle en était largement indépendante, y compris 
lorsque ses protagonistes s'appropriaient le discours de la bipolarité. En ce sens, elle nous fournit un cas idéal qui, par son antériorité, nous permet de tester la validité de l'ensemble des paradigmes actuels sur le " sens " des actions sociales.

4.P. L. Berger, Invitation to Sociology. A Humanistic Perspective. New York, Londres, Toronto, Anchor Books, 1963, p. 130.

5.M. Foucault, L'Archéologie du savoir, Paris, NRF, 1969, p. 12 et M. de Certeau, Histoire et Psychanalyse entre science et fiction, Paris, Gallimard, 1987, p. 22.

6.Ces associations étaient au nombre de 40.000 à la fin des années soixante-dix. On observe souvent que les autorités publiques demandent aux habitants de gecekondu: " où est donc ton association? " M. Heper, Türkiye'de Kent Göçmeni ve Bürokratik Örgütleri, Istanbul, Üçdal Nesriyat, 1983, p. 67.

7.Cf. pour l'analyse d'une communauté alévie, Altan Gökalp, Têtes Rouges et Bouches Noires, Paris, Société d'Ethnographie, 1980, pp. 63 et 67 ainsi qu'O. Türkdogan, AleviBektasi Kimligi, Sosyo-Antropolojik Arastirma, Istanbul, Timas, 1995.

8.Ce fait est loin d'être spécifique à la Turquie. Cf. pour trois autres pays du MoyenOrient, l'excellente étude de G. Denoeux, Urban Unrest in the Middle East: A Comparative Study of Informal Networks in Egypt, Iran and Lebanon, New York, State Univ. of New York, 1993.

9.Il s'agit du Parti de Justice et du Parti Populaire Républicain, dirigés respectivement par S. Demirel et B. Ecevit.

10.Les enquêtes de Metin Heper, réalisées dans la deuxième moitié des années soixante-dix, montrent clairement que le respect dû à l'Etat et l'image qu'on a de lui au quotidien sont dissociés. $82,1 \%$ des habitants d'un gecekondu, autrement dit, à la proximité immédiate du centre, " font confiance à l'Etat pour prendre les décisions les plus justes " (contre 13,2\%). Mais dès qu'il s'agit de savoir si l'Etat " risque de prendre une décision les endommageant ou endommageant leur quartier dans sa totalité " les réponses sont affirmatives à $65,1 \%$, et seulement à $19 \%$ négatives. M. Heper, op.cit. De même, dans les enquêtes de Metin Kazanci, à la question de " quelle serait votre attitude par rapport à une action ou un acte que vous croiriez utile pour les groupes à bas revenus, mais contraires aux lois écrites ou aux décrets ", seuls $11 \%$ répondent par : " j'essayerai de m'accorder à la loi ". Les autres se déclarent prêts à défier la loi. M. Kazanci, Halkla Iliskiler Açisindan Yönetim ve Yönetilenler, AÜSBFY, 1978, p. 191. 11.J. Leca, " Individualisme et citoyenneté ", in P. Birnbaum et J. Leca (dir.), Sur l'individualisme, Paris, FNSP, 1991, p. 159-209.

12.Le Parti Populaire Républicain a été créé par le fondateur de la République $M$. Kemal. Dans les années soixante-dix, il a tenté, sous l'impulsion de son nouveau chef B. Ecevit, de parvenir à une synthèse entre le kémalisme et la social-démocratie.

13.En effet, les clivages doctrinaux reflètent mal les dynamiques de la mobilisation qui restent largement segmentaires. Ainsi, la gauche puise essentiellement parmi les Alévis et les Kurdes, la droite essentiellement parmi les Turcs et Sunnites. Dans des régions mixtes, les Kurdes sunnites apportent leurs votes à l'extrême-droite.

14.Notamment les provinces kurdes et celles de l'Anatolie centrale.

15.Le nombre des gecekondu monte en flèche. En 1955 ils sont au nombre de 50.000 et abritent $4,7 \%$ de la population urbaine, en 1980 ils sont 1.150 .000 et abritent $26,1 \%$ de la population urbaine. Les estimations pour 1995 sont les suivantes : 2.200 .000 gecekondu abritant 34\% de la population urbaine. M. Sönmez, " Gecekonduyu Seçim Malzemesi Yapmayin ", Milliyet, 1.12.1995. 
16.Cf. entre autre O. Carlier, Entre Nation et Jihad. Histoire sociale des radicalismes algériens, Paris, FNSP, 1995.

17.Cf. pour cette notion, E. H. Erikson, Adolescence et crise. La Quête de l'Identité, Paris, Flammarion, 1978.

18.Idem.

19.Ainsi le PKK figure parmi les derniers acteurs entrés sur la scène politique. Il existe, sous une forme organisée, depuis la fin de 1977.

20.Ainsi, une bonne partie de la classe politique aujourd'hui est composée des acteurs " contestataires " de l'époque.

21.Milli Selamet Partisi (Parti du Salut National), dirigé par N. Erbakan, ancêtre du Parti de la Prospérité, la première formation du paysage politique turc actuel.

22.Ainsi A. Türkes, le dirigeant de l'extrême-droite expliquait que " celui qui contrôle les rues de ces deux grandes villes \{Istanbul et Ankara\}, s'emparera du pouvoir en Turquie ", Cité par Milliyet 76, Istanbul, Milliyet Yayinlari, 1977, p. 40.

23.J. B. Rule, Theories of Civil Violence, Berkeley, Los Angeles, Londres, Univ. of California Press, 1988, p. 211.

24.Le thème de la violence milicienne en Turquie a fait l'objet d'une recherche que nous avons présentée au CERI : " Le phénomène milicien : une composante de la violence politique en Turquie des années soixante-dix ". Une publication collective à venir rassemblera ce travail ainsi ceux d'Elizabeth Picard sur le Liban et de Xavier Bougarel sur la Bosnie.

25.A la question de Dogu Ergil (Türkiye'de Terör ve Siddet, Ankara, Turhan Kitabevi, 1980), " quelles sont les personnes dont l'" avis vous importe " : cf. annexe de la note 25. 26.Lors de ces élections le MHP (Parti d'Action Nationaliste) d'Alparslan Türkes parvient à multiplier par deux son score et obtient $6,4 \%$ des voix. Mais ce résultat constitue également le maximum de ce qu'il peut obtenir par la voie électorale, ce qui le pousse à privilégier la stratégie de conquête du pouvoir par la violence.

27.Jean Leca, " Algérie : Sur quelques aspects de la violence politique ", MaghrebMachrek, n¹41, 1991, p.105.

28. Le coup d'Etat a en effet suspendu les activités des partis politiques avant de les dissoudre.

29.L'expression est de l'intellectuel islamiste M. Dogan, Halka Karsi Demokrasi, Istanbul, Beyan Yayinlari, 1988.

30.Sur ce sujet, cf. J. B. Rule, op.cit.

31.Cf. pour la discussion de ce thème P. Saunders, Social Theory and Urban Question, Londres et New York, Routledge, 1993, pp. 39-51.

32.P. Berger, op.cit.

\section{RÉSUMÉS}

Hamit Bozarslan analyses the Turkish 1975-1980s crisis, which was both a political crisis and an economical recession. In this context, the confrontation between left-wing and right-wing radical groups generated a civil war climate to the point that the army massively intervened in 
1980. Though this crisis has long been analysed as a situation of chaos, progressively becoming independent from any control, the author insists on the actors' alliance or confrontation strategies in the deployment of violence. Violence is not the result of unpersonal and anonymous forces but results from political and social processes, which actors and interactions ought to be understood.

INDEX

Index géographique : Turquie

Mots-clés : crises politiques

Index chronologique : 1970 - 1980

\section{AUTEUR}

HAMIT BOZARSLAN

Chercheur au Centre Marc Bloch à Berlin. 